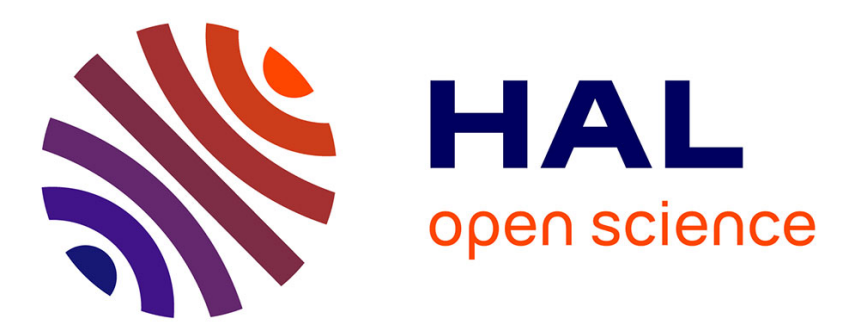

\title{
La microanalyse du soufre par l'observation de réactions nucléaires
}

\author{
R. Caplain, D. David, G. Béranger
}

\section{To cite this version:}

R. Caplain, D. David, G. Béranger. La microanalyse du soufre par l'observation de réactions nucléaires. Revue de Physique Appliquée, 1982, 17 (8), pp.441-445. 10.1051/rphysap:01982001708044100 . jpa00245020

\section{HAL Id: jpa-00245020 https://hal.science/jpa-00245020}

Submitted on 1 Jan 1982

HAL is a multi-disciplinary open access archive for the deposit and dissemination of scientific research documents, whether they are published or not. The documents may come from teaching and research institutions in France or abroad, or from public or private research centers.
L'archive ouverte pluridisciplinaire HAL, est destinée au dépôt et à la diffusion de documents scientifiques de niveau recherche, publiés ou non, émanant des établissements d'enseignement et de recherche français ou étrangers, des laboratoires publics ou privés. 
Classification

Physics Abstracts

25.10

\title{
La microanalyse du soufre par l'observation de réactions nucléaires
}

\author{
R. Caplain, D. David et G. Béranger \\ Université de Technologie, Département de Génie Mécanique, \\ Division des Matériaux, B.P. 233, 60206 Compiègne, France
}

(Reçu le 25 mai 1981, révisé le 22 février 1982, accepté le 6 mai 1982)

\begin{abstract}
Résumé. - La microanalyse nucléaire, déjà utilisée pour le dosage de nombreux éléments légers, a été appliquée à la recherche de l'isotope ${ }^{32} \mathrm{~S}$. Cette extension est justifiée par le rôle essentiel que joue fréquemment le soufre dans les mécanismes de corrosion. La réaction nucléaire ${ }^{32} \mathrm{~S}(\mathrm{~d}, \mathrm{p})^{33} \mathrm{~S}$ fournit sept pics, observables sur le spectre d'énergie des protons émis. Ces pics permettent d'effectuer, dans la plupart des cas, un dosage précis et quantitatif.

Les sections efficaces différentielles des pics correspondant aux états excités 7 et 32 ont été mesurées de $1100 \mathrm{keV}$ à $1450 \mathrm{keV}$ environ, ce qui a permis de définir les énergies de bombardement les plus appropriées au dosage d'échantillons épais.
\end{abstract}

\begin{abstract}
The nuclear microanalysis, already used for the dosage of numerous light elements, has been applied to the isotope ${ }^{32} \mathrm{~S}$ research. This extension is justified by the essential role that sulfur takes frequently in corrosion mechanisms. The nuclear reaction ${ }^{32} \mathrm{~S}(\mathrm{~d}, \mathrm{p}){ }^{33} \mathrm{~S}$ provides us with seven peaks that can be observable, which can permit, in most cases, a precise and quantitative dosage.

The differential cross sections for the peaks associated with the 7th and 32th excited states have been measured between about $1100 \mathrm{keV}$ and $1450 \mathrm{keV}$, which permit us to choise the most appropriated bombardment energies for dosage of thick samples.
\end{abstract}

Introduction. - Le soufre joue un rôle essentiel dans le comportement de nombreux métaux, notamment vis-à-vis de la corrosion. Il peut agir de l'extérieur, comme un des composants de l'environnement (corrosion atmosphérique, notamment). Ou bien, apparaissant en tant qu'impureté au sein du métal luimême, sous la forme d'une solution solide ou d'inclusions de sulfures, il est à l'origine de la formation de piqûres, puis d'une dégradation plus étendue. Il peut ainsi se combiner au nickel, au manganèse, au magnésium, au titane, au zirconium, entre autres. L'élaboration des alliages industriels à base de fer peut entraîner la formation de telles inclusions. Lorsque ces alliages sont soumis à un processus d'attaque électrochimique, un enrichissement en soufre de leur couche superficielle est susceptible de se produire, et il peut modifier complètement la résistance de l'alliage à la corrosion. Des travaux récents ont été consacrés au rôle du soufre dans les phénomènes de corrosion, notamment dans le cas du nickel et de ses alliages [1, 2].

La méthode de microanalyse par l'observation directe de réactions nucléaires est utilisée couramment pour le dosage de nombreux éléments légers, tels que l'oxygène, l'azote, le carbone ou le fluor. Dans certains cas, elle peut être appliquée à des isotopes d'un même élément ; c'est ainsi qu'on peut aisément distinguer ${ }^{16} \mathrm{O}$ et ${ }^{18} \mathrm{O}$, ou encore ${ }^{14} \mathrm{~N}$ et ${ }^{15} \mathrm{~N}$. Nous rappellerons seulement que le principe consiste à observer et analyser le spectre énergétique des particules émises par un échantillon lorsque celui-ci est bombardé par un faisceau de particules chargées monoénergétiques. Les particules incidentes sont généralement des deutons ou des protons; le flux émis est composé de protons et de particules alpha. Un exposé détaillé de cette méthode, qui est quantitative et non destructive, a été fait dans la référence [3]. Nous ne décrirons donc ici que les caractéristiques propres au dosage du soufre, que nous avons par ailleurs déjà rapidement mentionnées [4].

Parmi les autres techniques permettant de doser cet élément, citons la spectrométrie des électrons Auger, associée parfois à la diffraction des électrons lents [5, 6]. Cette méthode peut fournir des informations de nature chimique, sur quelques dizaines d'angströms de profondeur. L'exploitation quantitative est en revanche délicate, surtout sur les spectres ayant subi un traitement mathématique de dérivation [7].

La spectrométrie ESCA, quant à elle, fournit 
essentiellement des informations sur les liaisons chimiques, la profondeur accessible à chaque mesure n'excédant pas $50 \AA$ [7]. Toutefois, l'utilisation simultanée d'un système d'abrasion ionique permet de dépasser cette limite, avec les réserves habituelles concernant alors l'interprétation des résultats. On arrive ainsi à tracer des profils s'étendant sur quelques milliers d'angströms [9].

Enfin, des mesures quantitatives peuvent être effectuées au moyen du traceur radioactif ${ }^{35} S$ [10]. Cet isotope est instable, mais sa période est suffisante pour que l'expérimentation soit possible dans de bonnes conditions. Celle-ci nécessite cependant la préparation d'échantillons marqués, dont la durée de vie utile est limitée [11]. Le recours à une méthode d'analyse permettant de doser l'isotope naturel ${ }^{32} \mathrm{~S}$ est donc nécessaire pour l'étude d'échantillons ne pouvant être préparés dans un laboratoire. C'est le cas, par exemple, des matériaux exposés dans les stations d'essais qui étudient la corrosion d'origine atmosphérique. En outre, la possibilité de doser séparément deux isotopes d'un même élément augmente considérablement le champ des expérimentations possibles (études d'autodiffusion, par exemple).

Signalons enfin que la microanalyse nucléaire permet, non seulement d'observer des couches minces de quelques dizaines d'angströms, mais aussi de mesurer des profils de concentration sur des profondeurs qui peuvent atteindre 1 à $2 \mu \mathrm{m}$, sans qu'il soit nécessaire d'effectuer des abrasions.
1. Conditions expérimentales. - Nous avons exposé, lors de la description des conditions d'analyse de l'azote, quelles conditions devaient satisfaire les réactions nucléaires utilisées [12]. Ces conditions sont ici remplies de façon satisfaisante par la réaction ${ }^{32} \mathrm{~S}(\mathrm{~d}, \mathrm{p}){ }^{33} \mathrm{~S}$; le tableau $\mathrm{I}$ en indique les principales caractéristiques, pour les états d'excitation dont nous avons pu observer les pics sur le spectre énergétique des particules émises. Les relations de la cinématique permettent de calculer les énergies de ces particules, avant le film de mylar placé devant le détecteur. La fonction de transfert de ce film permet ensuite de déterminer les valeurs de l'énergie au niveau du détecteur lui-même. Ces pics sont rangés par ordre d'énergie décroissante, tels qu'ils apparaissent sur la figure 1 . Il est à remarquer que, sur cette figure, les pics situés au-dessus du canal 105 sont agrandis en ordonnée d'un facteur 10. Le tableau mentionne les mêmes informations pour l'oxygène, l'azote et le carbone, en raison de l'abondance de ces éléments dans le milieu naturel, et donc de la forte probabilité de les observer à la surface des échantillons.

Signalons également l'existence de la réaction ${ }^{32} \mathrm{~S}(\mathrm{~d}, \alpha)^{30} \mathrm{P}$. Le pic qu'elle engendre n'est pas utilisable dans nos conditions expérimentales en raison de son faible rendement et de sa superposition partielle aux pics voisins.

1.1 DESCRIPTION D'UN SPECTRE TYPIQUE D'AMPLITUDE. - La figure 1 représente un spectre caracté-

Tableau I.

\begin{tabular}{|c|c|c|c|c|c|}
\hline Réaction & Etat & $\begin{array}{c}\text { Energie } \\
\text { avant mylar } \\
(\mathrm{MeV})\end{array}$ & $\begin{array}{c}\text { Energie } \\
\text { après mylar } \\
(\mathrm{MeV})\end{array}$ & Canal & $\underset{(\mathrm{MeV})}{Q}$ \\
\hline - & - & - & - & - & - \\
\hline${ }^{32} \mathrm{~S}(\mathrm{~d}, \mathrm{p})^{33} \mathrm{~S}$ & $32 *$ & 1,678 & 1,12 & 12 & 0,707 \\
\hline${ }^{16} \mathrm{O}(\mathrm{d}, \mathrm{p}){ }^{17} \mathrm{O}$ & $*$ & 1,790 & 1,26 & 16 & 1,048 \\
\hline${ }^{14} \mathrm{~N}(\mathrm{~d}, \mathrm{p})^{15} \mathrm{~N}$ & $5^{*}$ & 1,963 & 1,47 & 21 & 1,305 \\
\hline${ }^{14} \mathrm{~N}(\mathrm{~d}, \mathrm{p}){ }^{15} \mathrm{~N}$ & $4^{*}$ & 2,096 & 1,64 & 26 & 1,455 \\
\hline $\mathrm{D}(\mathrm{d}, \mathrm{p}) \mathrm{T}$ & F & 2,306 & 1,88 & 32 & 4,033 \\
\hline${ }^{32} \mathrm{~S}(\mathrm{~d}, \alpha)^{30} \mathrm{P}$ & $F$ & 4,952 & 2,00 & 35 & 4,890 \\
\hline${ }^{16} \mathrm{O}(\mathrm{d}, \mathrm{p})^{17} \mathrm{O}$ & $\mathrm{F}$ & 2,573 & 2,18 & 39 & 1,919 \\
\hline${ }^{32} \mathrm{~S}(\mathrm{~d}, \mathrm{p})^{33} \mathrm{~S}$ & $17^{*}$ & 2,588 & 2,20 & 40 & 1,670 \\
\hline${ }^{14} \mathrm{~N}(\mathrm{~d}, \mathrm{p}){ }^{15} \mathrm{~N}$ & $3^{*}$ & 2,835 & 2,47 & 47 & 2,285 \\
\hline${ }^{12} \mathrm{C}(\mathrm{d}, \mathrm{p})^{13} \mathrm{C}$ & $\mathrm{F}^{\circ}$ & 3,129 & 2,80 & 54 & 2,719 \\
\hline${ }^{32} \mathrm{~S}(\mathrm{~d}, \mathrm{p})^{33} \mathrm{~S}$ & $8^{*}$ & 3,455 & 3,17 & 64 & 2,584 \\
\hline${ }^{14} \mathrm{~N}(\mathrm{~d}, \mathrm{p}){ }^{15} \mathrm{~N}$ & $*$ et $* *$ & $3,776 / 3,746$ & 3,48 & 73 & $\begin{array}{l}3,335 \\
3,305\end{array}$ \\
\hline${ }^{32} \mathrm{~S}(\mathrm{~d}, \mathrm{p})^{33} \mathrm{~S}$ & $7 *$ & 4,036 & 3,76 & 80 & 3,194 \\
\hline${ }^{32} \mathrm{~S}(\mathrm{~d}, \mathrm{p})^{33} \mathrm{~S}$ & $4^{*}$ & 4,374 & 4,13 & 87 & 3,549 \\
\hline${ }^{14} \mathrm{~N}(\mathrm{~d}, \alpha)^{12} \mathrm{C}$ & $*$ & 6,739 & 4,60 & 101 & 9,146 \\
\hline${ }^{32} \mathrm{~S}(\mathrm{~d}, \mathrm{p})^{33} \mathrm{~S}$ & $3^{*}$ & 4,904 & 4,67 & 103 & 4,105 \\
\hline${ }^{32} S(d, p)^{33} S$ & $2^{*}$ & 5,235 & 5,01 & 111 & 4,453 \\
\hline${ }^{34} \mathrm{~S}(\mathrm{~d}, \mathrm{p})^{35} \mathrm{~S}$ & $\mathrm{~F}$ & 5,549 & 5,34 & 120 & 4,757 \\
\hline${ }^{32} \mathrm{~S}(\mathrm{~d}, \mathrm{p})^{33} \mathrm{~S}$ & $*$ & 6,309 & 6,11 & 139 & 5,577 \\
\hline${ }^{14} \mathrm{~N}(\mathrm{~d}, \alpha)^{12} \mathrm{C}$ & F & 9,880 & 8,38 & 194 & 13,579 \\
\hline
\end{tabular}




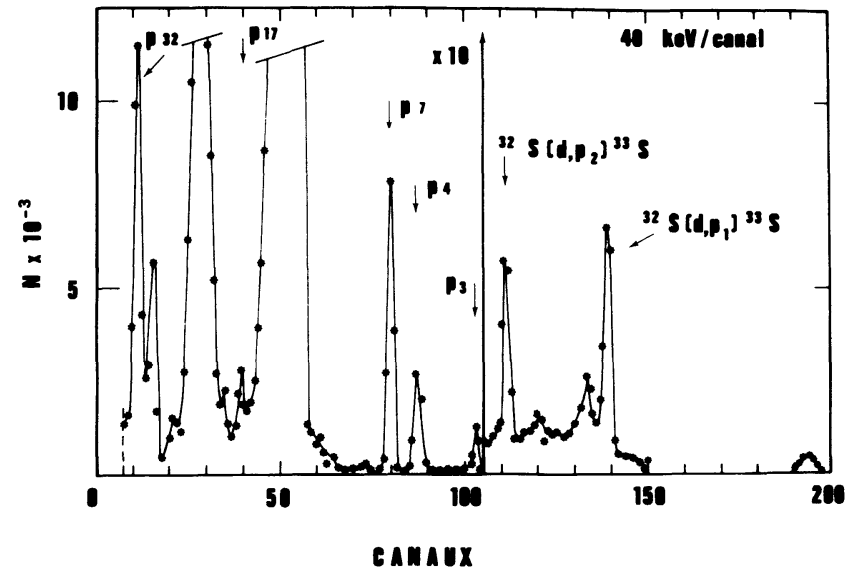

Fig. 1. - Spectre correspondant à un dépôt de soufre de $2000 \AA$ d'épaisseur, bombardé par des deutons de $1200 \mathrm{keV}$. Observation à $150^{\circ}$, absorbant en mylar de $19 \mu \mathrm{m}$.

[A typical energy spectrum from the deuteron bombardment of a $2000 \AA$ thick sulfur target on nickel backing. $E_{0}=1200 \mathrm{keV}, 19 \mu \mathrm{m}$ mylar, $150^{\circ} \mathrm{C}$.]

ristique, obtenu en bombardant avec des deutons de $1200 \mathrm{keV}$ une cible de sulfure de nickel de (2 $000 \pm 500) \AA$ d'épaisseur, déposée par évaporation sur un support de nickel. L'angle d'observation par rapport au faisceau incident est de $150^{\circ}$ (repère du laboratoire). Le détecteur, d'une surface de $3 \mathrm{~cm}^{2}$, est placé à $5 \mathrm{~cm}$ de l'échantillon. Un film de mylar de $19 \mu \mathrm{m}$, placé devant le détecteur, arrête les particules rétrodiffusées et évite la saturation de la chaîne de mesure. La pression résiduelle dans l'enceinte d'analyse est voisine de $10^{-5}$ torr.

Dans ces conditions, les seuls pics bien visibles sont ceux des états excités $1,2,3,4,7,17$ et 32 de la réaction ${ }^{32} \mathrm{~S}(\mathrm{~d}, \mathrm{p}){ }^{33} \mathrm{~S}$. Avec les réglages utilisés, ils apparaissent respectivement dans les canaux 139, $111,103,87,80,40$ et 12 , par ordre d'énergie décroissante.

Le pic de l'état fondamental de la réaction ${ }^{32} \mathrm{~S}(\mathrm{~d}, \mathrm{p}){ }^{33} \mathrm{~S}$ n'est pas visible sur le spectre de la figure 1 . En effet, avec une valeur $Q$ de $6,418 \mathrm{MeV}$, ce pic correspond à des protons de $6,94 \mathrm{MeV}$ qui ne sont pas arrêtés par le détecteur de particules. La zone sensible de celui-ci, épaisse de $300 \mu \mathrm{m}$ pour une polarisation de $120 \mathrm{~V}$, ne permet de détecter que des protons d'une énergie maximale de 5,2 MeV. Au-delà, les pics sont remplacés par des bosses arrondies, dont on peut modifier l'emplacement dans le spectre en jouant sur la polarisation du détecteur [12].

La partie grossie 10 fois du spectre montre, légèrement détachés du bruit de fond environnant, les pics des deux premiers états excités de ${ }^{32} S(d, p){ }^{33} S$. Le pic de la réaction ${ }^{34} \mathrm{~S}(\mathrm{~d}, \mathrm{p}){ }^{35} \mathrm{~S}$ apparaît entre eux, et sa faible amplitude est évidemment due, au moins en partie, à la faible abondance isotopique de ${ }^{34} \mathrm{~S}$. Un pic de faible intensité, mal identifié, apparaît également dans cette zone (canal 133). Il n'est pas exclu qu'il soit imputable à une réaction nucléaire sur le chlore, l'isotope marqué ${ }^{35} \mathrm{~S}$ se transformant spontanément en ${ }^{35} \mathrm{Cl}$. En effet, le $\mathrm{H}_{2} \mathrm{~S}$ gazeux utilisé pour préparer le sulfure de nickel constituant l'échantillon comprend à la fois les isotopes ${ }^{32} \mathrm{~S}$ et ${ }^{35} \mathrm{~S}$.

On observe ensuite, par ordre d'énergie décroissante, le pic du $3^{\mathrm{e}}$ état excité, dans le canal 103. Son utilisation est à éviter, car il est superposé au pic de la réaction ${ }^{14} \mathrm{~N}(\mathrm{~d}, \alpha)^{12} \mathrm{C}^{*}$. En revanche, les pics des états excités 4 et, surtout, 7 , présentent un fort taux de comptage, très supérieur à celui des états 1 et 2 , et ils sont bien détachés des pics voisins.

Le pic de la réaction ${ }^{12} \mathrm{C}(\mathrm{d}, \mathrm{p}){ }^{13} \mathrm{C}$ est particulièrement important pour l'échantillon que nous avons étudié. Il contrarie donc l'observation des petits pics voisins. Il en est de même du grand pic qui apparaît vers le canal 28 et qui pourrait correspondre, dans l'échelle des énergies, à la réaction $\mathrm{D}(\mathrm{d}, \mathrm{p}) \mathrm{T}$. Il se peut toutefois que ce pic soit la résultante de plusieurs réactions, ce que permettront seules de déterminer des expériences ultérieures, faites avec des échantillons de nature différente.

Toutefois, ce pic est observable sur du nickel poli, de même origine que celui utilisé pour la préparation des échantillons de sulfure. Il est donc vraisemblablement imputable à des impuretés contenues dans ce métal et, par suite, n'a pas de rapport direct avec l'analyse nucléaire du soufre.

Précisons que l'identification des différents pics, telle que nous l'exposons ici, repose sur la concordance énergie mesurée/énergie calculée, qui est réalisée à \pm 1 canal près. Elle offre donc une bonne probabilité d'exactitude, mais demande à être confirmée par des observations complémentaires, réalisées sur divers types d'échantillons et pour plusieurs énergies de bombardement.

Pour terminer l'examen du spectre présenté ici, mentionnons le pic du $32^{e}$ état excité de ${ }^{32} \mathrm{~S}(\mathrm{~d}, \mathrm{p})^{33} \mathrm{~S}$, qui apparaît dans le canal 12. Son taux de comptage est important, mais il est voisin du pic de la réaction ${ }^{16} \mathrm{O}(\mathrm{d}, \mathrm{p})^{17} \mathrm{O}$ (1 ${ }^{\text {er }}$ état excité), ce quì peut créer des difficultés pour l'analyse d'échantillons comportant à la fois du soufre et de l'oxygène.

1.2 Mesure Des SECTIONS EFFICACES DIFFÉRENTIELLES. - Nous avons mesuré la section efficace différentielle du pic issu de la réaction ${ }^{32} \mathrm{~S}(\mathrm{~d}, \mathrm{p})^{33} \mathrm{~S}$ (32e état excité), de $1140 \mathrm{keV}$ à $1450 \mathrm{keV}$, pour un angle d'observation de $150^{\circ}$ dans le repère du laboratoire (Fig. 2). En effet, bien qu'il apparaisse à une énergie assez faible et soit, de ce fait, relativement mal isolé, ce pic présente un fort taux de comptage et permet donc la réalisation de mesures rapides. Ce point revêt toute son importance si on considère que les sections efficaces sont ici de quelques dixièmes de millibarn/stéradian, et sont donc nettement plus faibles que dans le cas de l'azote et, surtout, de l'oxygène [3]. La courbe présente une zone croissante de $1210 \mathrm{keV}$ à $1400 \mathrm{keV}$, encadrée par deux domaines 


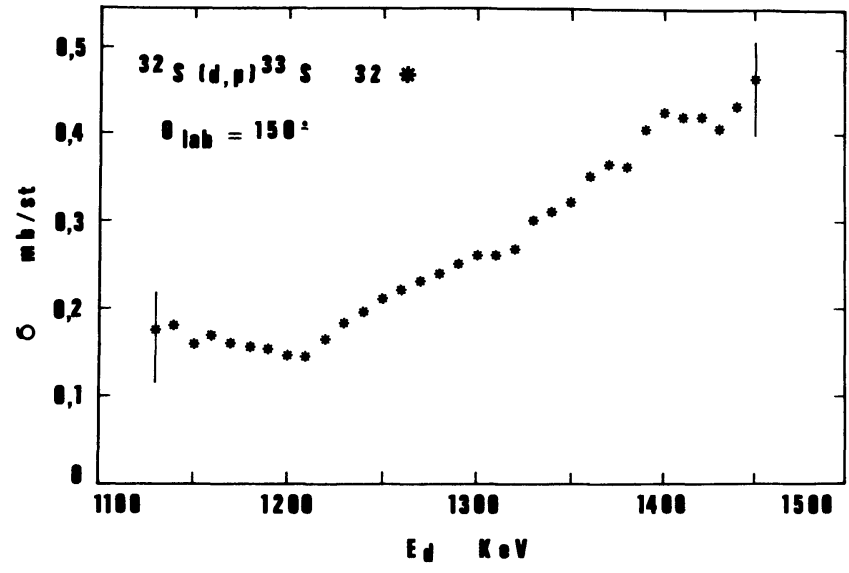

Fig. 2. - Section efficace différentielle de la réaction ${ }^{32} \mathrm{~S}(\mathrm{~d}, \mathrm{p}){ }^{33} \mathrm{~S}$ (32e état excité).

[Differential cross section of the ${ }^{32} \mathrm{~S}(\mathrm{~d}, \mathrm{p}){ }^{33} \mathrm{~S}$ reaction (32th excited state).]

où la variation est faible, et qui peuvent être assimilés à des plateaux. Le $1^{\text {er }}$ plateau s'étend de 1140 à $1210 \mathrm{keV}$ environ. Cette étendue de $70 \mathrm{keV}$ correspond, pour des deutons de $1200 \mathrm{keV}$, à une excursion possible en profondeur de l'ordre de $0,5 \mu \mathrm{m}$ dans le cas du nickel (pouvoir d'arrêt de $154,5 \mathrm{MeV} . \mathrm{cm}^{2} / \mathrm{g}$ ) [13]. Le $2^{\mathrm{e}}$ plateau s'étend de 1400 à $1440 \mathrm{keV}$ environ. Il correspond à une excursion de $0,3 \mu \mathrm{m}$ pour le nickel $\left(145,2 \mathrm{MeV} \cdot \mathrm{cm}^{2} / \mathrm{g}\right)$. Les valeurs de ces excursions en profondeur dépendent fortement du matériau considéré. Ainsi, elles sont respectivement de $0,9 \mu \mathrm{m}$ et $0,5 \mu \mathrm{m}$ dans le cas du titane.

Nous avons volontairement limité cette courbe au domaine indiqué ci-dessus car, aux énergies inférieures à $1140 \mathrm{keV}$, le pic tend à se confondre avec celui de la réaction ${ }^{16} \mathrm{O}(\mathrm{d}, \mathrm{p}){ }^{17} \mathrm{O}^{*}$; aux énergies supérieures à $1450 \mathrm{keV}$, c'est le pic de la réaction ${ }^{14} \mathrm{~N}(\mathrm{~d}, \mathrm{p}){ }^{15} \mathrm{~N}^{6 *}$ qui devient trop proche.

Nous avons également mesuré, dans les mêmes conditions, la section efficace différentielle du pic ${ }^{32} \mathrm{~S}(\mathrm{~d}, \mathrm{p}){ }^{33} \mathrm{~S}$ (7e état excité), de $1050 \mathrm{keV}$ à $1380 \mathrm{keV}$ (Fig. 3). La limite supérieure de ce domaine d'énergie est imposée par le début de la superposition avec le pic ${ }^{12} \mathrm{C}(\mathrm{d}, \mathrm{p}){ }^{13} \mathrm{C}$. La limite inférieure correspond à un taux de comptage qui devient trop faible. Entre ces limites, la section efficace croît régulièrement, une certaine stabilisation apparaissant toutefois de 1340 à $1380 \mathrm{keV}$. Sa valeur est alors comparable à celle mesurée, aux mêmes énergies, pour le pic du $32^{\mathrm{e}}$ état excité.

Nous n'avons pas mesuré la section efficace du pic correspondant au $4 \mathrm{e}$ état excité car, en tout état de cause, il apparaît préférable d'utiliser le pic du $7 \mathrm{e}$ état : emplacement voisin dans l'échelle des énergies et taux de comptage plus élevé.

1.3 RÉACTIONS PARASITES. - Le tableau II indique les valeurs des énergies de réaction $Q$, pour les réactions

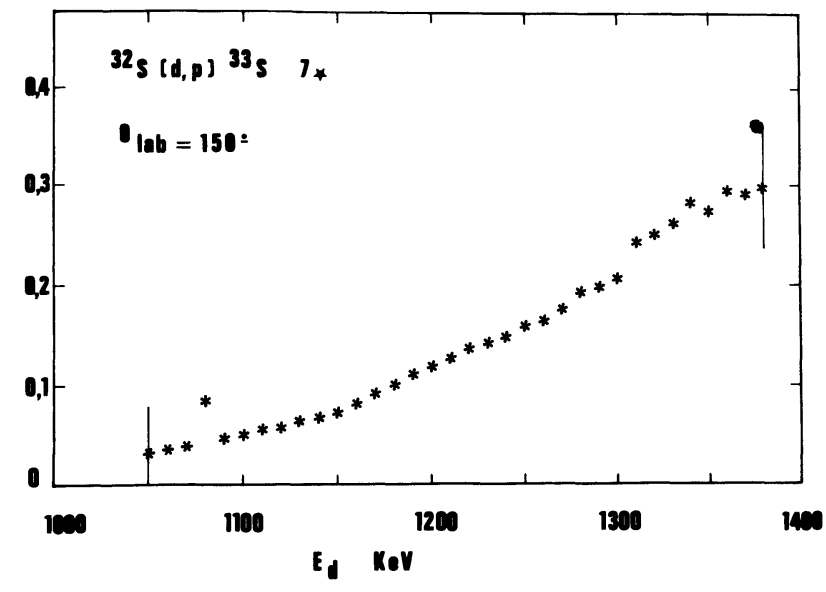

Fig. 3. - Section efficace différentielle de la réaction ${ }^{32} \mathrm{~S}(\mathrm{~d}, \mathrm{p}){ }^{33} \mathrm{~S}$ (7e état excité).

[Differential cross section of the ${ }^{32} \mathrm{~S}(\mathrm{~d}, \mathrm{p}){ }^{33} \mathrm{~S}$ reaction (7th excited state).]

Tableau II. - Réactions (d, p).

$\begin{array}{lclc}\text { Elément } & Q_{0}(\mathrm{MeV}) & \text { Elément } & Q_{0}(\mathrm{MeV}) \\ - & - & - & - \\ { }^{10} \mathrm{~B} & 9,237 & { }^{9} \mathrm{Be} & 4,585 \\ { }^{25} \mathrm{Mg} & 8,873 & { }^{32} \mathrm{~S}\left(\mathrm{p}_{2}\right) & 4,453 \\ { }^{14} \mathrm{~N}\left(\mathrm{p}_{0}\right) & 8,615 & { }^{19} \mathrm{~F} & 4,379 \\ { }^{29} \mathrm{Si} & 8,390 & { }^{30} \mathrm{Si} & 4,367 \\ { }^{32} \mathrm{~S} & 6,418 & { }^{26} \mathrm{Mg} & 4,212 \\ { }^{28} \mathrm{Si} & 6,253 & { }^{32} \mathrm{~S}\left(\mathrm{p}_{4}\right) & 3,549 \\ { }^{13} \mathrm{C} & 5,947 & { }^{32} \mathrm{~S}\left(\mathrm{p}_{7}\right) & 3,194 \\ { }^{31} \mathrm{P} & 5,712 & { }^{12} \mathrm{C} & 2,719 \\ { }^{17} \mathrm{O} & 5,842 & { }^{16} \mathrm{O} & 1,919 \\ { }^{32} \mathrm{~S}\left(\mathrm{p}_{1}\right) & 5,577 & { }^{18} \mathrm{O} & 1,731 \\ { }^{27} \mathrm{Al} & 5,499 & { }^{14} \mathrm{~N}\left(\mathrm{p}_{5}\right) & 1,305 \\ { }^{24} \mathrm{Mg} & 5,106 & { }^{11} \mathrm{~B} & 1,138 \\ { }^{6} \mathrm{Li} & 5,027 & { }^{32} \mathrm{~S}\left(\mathrm{p}_{32}\right) & 0,707 \\ { }^{23} \mathrm{Na} & 4,734 & { }^{15} \mathrm{~N} & 0,267\end{array}$

induites par les deutons sur les noyaux légers, avec. émission de protons. Si on tient compte de la faible proportion, dans les éléments naturels, de certains des isotopes mentionnés $\left({ }^{17} \mathrm{O},{ }^{13} \mathrm{C},{ }^{25} \mathrm{Mg}\right.$ ), on voit que le pic du premier état excité peut être brouillé par des réactions sur le phosphore, le silicium, l'azote ou le bore. Pour le pic du $2^{\mathrm{e}}$ état excité, s'ajoutent à ces éléments le béryllium, le sodium, le lithium, le magnésium et l'aluminium. La plupart de ces éléments sont cependant assez peu répandus à l'état naturel, ce qui restreint la probabilité de les voir perturber un spectre.

La probabilité d'observation de réactions parasites augmente quand les valeurs de $Q$ diminuent, mais l'existence de 7 pics caractéristiques du soufre est de nature à permettre, la plupart du temps, l'obtention de résultats satisfaisants. De même, on ne peut exclure toute possibilité d'interférences avec des réactions du type $(\mathrm{d}, \alpha)$. Il faut remarquer à ce sujet que les -particules alpha sont beaucoup plus sensibles que les protons à une modification de l'épaisseur du film de 
mylar placé devant le détecteur. En jouant sur cette propriété et sur le choix de l'énergie de bombardement, on est donc à même de résoudre la plupart des problèmes qui peuvent se poser.

2. Cibles de référence. - Le spectre représenté par la figure 1 a été obtenu sur un échantillon de nickel à $99,999 \%$, préparé à l'Ecole Nationale Supérieure de Chimie de Paris, dans le laboratoire du Professeur Oudar. Cet échantillon a subi un polissage mécanique, suivi d'un polissage électrolytique dans $\mathrm{H}_{2} \mathrm{SO}_{4}$ dilué. Il a été recuit $2 \mathrm{~h}$ à $700^{\circ} \mathrm{C}$ en atmosphère d'hydrogène purifié, aux fins de réduction de l'oxyde superficiel et recristallisation. Il a subi ensuite une sulfuration en atmosphère $\mathrm{H}_{2} \mathrm{~S} / \mathrm{H}_{2}$, à $380{ }^{\circ} \mathrm{C}$ durant $20 \mathrm{~min}$. La pression d'hydrogène était de 200 torr, et le rapport des pressions partielles $\mathrm{H}_{2} \mathrm{~S} / \mathrm{H}_{2}$ de $8 \times 10^{-4}$. Le $\mathrm{H}_{2} \mathrm{~S}$ est marqué en ${ }^{35} \mathrm{~S}$, afin de permettre un dosage quantitatif du dépôt par comptage de la radioactivité. Dans ces conditions, le support de nickel se trouve revêtu d'une couche de sulfure $\mathrm{Ni}_{3} \mathrm{~S}_{2}$, épaisse de $(2000 \pm 500) \AA[10,14,15]$.

Une telle cible mince convient particulièrement pour l'obtention de spectres typiques, permettant l'identification des différents pics et la mesure des sections efficaces différentielles.

Signalons cependant que nous avons parfois observé des anomalies dans la forme des spectres, et que celles-ci pourraient provenir de modifications des caractéristiques des échantillons produites par le faisceau incident. Il est possible d'éviter ce risque, dans une certaine mesure, en déplaçant le point d'impact du faisceau. Par ailleurs, ce point n'a pas laissé de traces visibles sur les échantillons après des bombardements d'une durée cumulée de plusieurs heures. Il ne sera possible d'acquérir une certitude à ce propos que par la multiplication des analyses, sur des échantillons de natures différentes.
3. Conclusion. - L'analyse du soufre par observation de réactions nucléaires est réalisable dans des conditions satisfaisantes, du fait de l'existence de 7 pics suffisamment isolés et détachés du bruit de fond pour permettre un comptage significatif. Toutefois, deux seulement d'entre eux ont une section efficace suffisante pour que les mesures soient réalisables dans de bonnes conditions. Dans la plupart des cas, on pourra utiliser le pic du $32^{e}$ état excité de ${ }^{32} \mathrm{~S}(\mathrm{~d}, \mathrm{p})^{33} \mathrm{~S}$, soit avec une énergie de bombardement de $1210 \mathrm{keV}$, soit avec une énergie de $1440 \mathrm{keV}$; dans ce dernier cas, le rendement sera amélioré d'un facteur 2 . Si des réactions parasites interfèrent avec ce pic, il restera possible d'observer le 7 e état excité de la même réaction, à $1380 \mathrm{keV}$. Les diverses valeurs de l'énergie du faisceau incident correspondent à des plateaux des courbes de section efficace différentielle; elles sont donc adaptées à l'analyse d'échantillons épais. Nos mesures n'ont pas permis d'observer des résonances fines, mais le pas de $10 \mathrm{keV}$ utilisé n'était pas assez serré pour acquérir une certitude à ce sujet. Des mesures complémentaires sont donc en cours, dans le but d'améliorer la définition des courbes de section efficace.

Remerciements. - Ce travail a été effectué au sein de l'Equipe de Recherche Associée au C.N.R.S. no 910.

Les auteurs remercient Monsieur G. Amsel, Directeur de Recherche, qui a permis la réalisation des mesures nucléaires dans le cadre de la R.C.P. $\mathrm{n}^{\circ} 157$ du C.N.R.S.

Ils remercient également Monsieur P. Marcus, Chargé de Recherche au C.N.R.S., membre du laboratoire de Monsieur le Professeur Oudar (Ecole Nationale Supérieure de Chimie de Paris), qui a bien voulu se charger de la préparation des cibles de sulfure.

Ce travail a bénéficié du soutien de la D.G.R.S.T. (action Matériaux-Corrosion $\mathrm{n}^{\circ}$ 81.P.0778).

\section{Bibliographie}

[1] Marcus, P., Oudar, J. et Olefjord, I., Mater. Sci. Eng. 42 (1980) 191.

[2] Colin, J. P., Thèse, Compiègne (1980).

[3] Amsel, G., Nadai, J. P., D'Artemare, E., David, D., Girard, E. and Moulin, J., Nucl. Instrum. Methods 92 (1971) 481.

[4] David, D., Cremery, P., Garcia, E. A., Béranger, G., J. Radioanal. Chem. 55 (1980) 345.

[5] Chuan C. Chang, Surf. Sci. 25 (1971) 53.

[6] Burggraf, C., Carriere, B. et Goldsztaub, S., Revue Phys. Appl. 11 (1976) 13.

[7] TOusSeT, J., J. Frott. Indust. 4 (1979) 1.

[8] Scheibner, E. J. et Tharp, L. N., Surf. Sci. 8 (1967) 247.
[9] Herglotz, H. K. and Suchan, H. L., Adv. Colloid Interface Sci. 5 (1975) 79.

[10] Oudar, J., C.R. Hebd. Séan. Acad. Sci. 249 (1959) 91.

[11] Endt, P. M. and VAN DER LeUn, C., Nucl. Phys. A 214 (1973).

[12] Amsel, G., David, D., Revue Phys. Appl. 4 (1969) 383.

[13] Williamson, C. F., Boujot, J. P. et Picard, J., Rapport C.E.A.-R 3042 (1966).

- [14] Benard, J., Oudar, J. et Cabane-Brouty, F., Surf. Sci. 3 (1965) 359.

[15] Marcus, P., Thèse, Paris (1979). 